\title{
PPIs shown to exacerbate NSAID injury in rats through dysbiosis
}

PPIs significantly exacerbate NSAIDinduced small intestinal damage, and such exacerbation is linked to marked shifts in the intestinal microbiota, so reports a study published in Gastroenterology.

PPIs are widely co-prescribed with NSAIDs to prevent gastrointestinal injury. Previous data suggesting that intestinal injury is observed in patients on PPIs and NSAIDs, and that PPIs can lead to small bowel bacterial overgrowth, encouraged Wallace and colleagues to examine whether PPIs could actually worsen NSAID enteropathy. If so, the group wanted to determine whether this exacerbation was due to changes in small intestinal bacteria.

Rats were given naproxen or celecoxib plus omeprazole or lansoprazole.

Intestinal damage was assessed directly and by hematocrit scoring. The intestinal microbiota was assessed by use of gel electrophoresis and quantitative PCR.
NSAID-related intestinal ulceration and bleeding was significantly increased by PPI use, and populations of bifidobacteria and Actinobacteria were reduced by $\sim 80 \%$. Colonization of germ-free mice with intestinal microbiota from PPI-treated rats increased the severity of NSAID damage. The intestinal damage could be prevented by restoring bifidobacteria levels.

"These results are consistent with clinical observations and should trigger a careful re-evaluation of the widespread practice of co-prescribing NSAIDs and PPIs," says John Wallace, corresponding author. Clinical trials are now warranted to investigate this concept further.

Rachel Thompson

Original article Wallace, J. L. et al. Proton pump inhibitors exacerbate NSAID-induced small intestinal injury by inducing dysbiosis. Gastroenterology doi:10.1053/ j.gastro.2011.06.075 